\title{
Improved Estimation of Radiated Fields of Unintentional Radiators by Correction of the Impedance Mismatch Between a Transverse Electromagnetic Cell and a Hybrid Coupler
}

\author{
Raul Blecic, Student Member, IEEE, Hrvoje Stimac, Student Member, IEEE, Renaud Gillon, Member, IEEE, \\ Bart Nauwelaers, Senior Member, IEEE and Adrijan Baric, Senior Member, IEEE
}

\begin{abstract}
This work is supported in part by the Croatian Science Foundation (HRZZ) within the project Advanced design methodology for switching DC-DC converters.

R. Blecic is with ESAT-TELEMIC (KU Leuven, B-3001 Heverlee, Bel-gium) and Faculty of Electrical Engineering and Computing (FER), University of Zagreb, 10000 Zagreb, Croatia, as a double degree PhD student.

H. Stimac and A. Baric are with Faculty of Electrical Engineering and Computing (FER), University of Zagreb, 10000 Zagreb, Croatia.

R. Gillon is with ON Semiconductor, Westerring 15, 9700 Oudenaarde, Belgium.

B. Nauwelaers is with KU Leuven, B-3001 Heverlee, Belgium.
\end{abstract}

\begin{abstract}
Radiated fields of unintentional radiators can be estimated from the measurements performed by a transverse electromagnetic cell. A hybrid coupler connected to the outputs of the transverse electromagnetic cell separates the electric and magnetic coupling, which allows to calculate the generated far-field radiation consistently. However, the transverse electro-magnetic cell and the hybrid coupler are mismatched which introduces an error in the measured results. The error of up to $8.5 \mathrm{~dB}$ is obtained in the measurements of the small loop. The impedance mismatch is dominantly a consequence of the phase shift which is related to the propagation of the signal through the measurement setup and which is present even if all elements of the measurement setup are ideal. The methodology for correcting the measured results is proposed and it is validated on the measurements of the small loop. The methodology is applied to the measurements of two synchronous buck converters and their radiated fields are estimated.
\end{abstract}

\section{INTRODUCTION}

Radiated fields generated by unintentional radiators can violate the limits specified by the regulations [1].The compliance with the regulations is verified by measuring a device at an open-area test site (OATS) or in a semi-anechoic chamber (SAC) [1]. Alternatively, the radiated fields can be estimated from the measurements performed in a transverse

electromagnetic cell (TEM) or in a gigahertz TEM (GTEM) cell [2]-[5]. The estimated radiated fields are compared to the radiated fields measured at the OATS in [2], [6]-[8] and in the $\mathrm{SAC}$ in [9]-[11] and a reasonable level of correlation is obtained.

The main challenge when using a TEM or GTEM cell is the calculation of the radiated fields from the measured data. Typically, a device under test (DUT) is approximated as electrically small and its radiation characteristics are described by three orthogonal electric and three orthogonal magnetic dipole moments. The dipole moments can be extracted from the measurements of the DUT at different orientations relative to the septum of the TEM or GTEM cell [2]-[5].
The radiated fields can then be calculated by combining the contributions of each of the extracted dipole moments [2].An algorithm for extraction of the dipole moments from nine measurements performed in a GTEM cell is proposed in [2]. However, it is shown in [5] that the dipole moments extracted by this algorithm depend on the starting orientation of the DUT inside the GTEM cell. A least-square algorithm which simultaneously fits the dipole moments to a large number of measurements performed at different orientations relative to the septum of the GTEM cell and which gives a consistent set of dipole moments is proposed in the same paper. However, performing a large number of measurements is impractical and time consuming. A TEM cell offers an advantage over a GTEM cell as it allows to separate the contribution of the electric and magnetic dipole moments of the DUT by using the phase information of its two output signals (a GTEM cell has one output). A hybrid coupler can be used to perform the separation [3], [4]. Only six measurements are needed to extract a consistent set of three orthogonal electric and three orthogonal magnetic dipole moments. The measurement setup based on a TEM cell and a hybrid coupler has been used recently in [9] to extract the radiation model of an integrated circuit, in [12] to characterize a synchronous buck converter, in [13] to estimate the maximum radiation from printed circuit boards (PCBs) with cables due to the magnetic field coupling, in [14] to characterize microstrip traces and a square patch, and in [15] to characterize a microstrip trace. However, none of these papers deal with the mismatch between the TEM cell and the hybrid coupler. While it may be difficult to identify the impact of the mismatch in the measurements of complex unintentional radiators as their radiation characteristics are unknown, the impact can be seen in the measurements of structures with the known radiation characteristics. Such measurements are presented in [14], [15] in which the impact of the mismatch is seen as the ringing of the measured coupled voltage.

In this paper, the impact of the impedance mismatch be-tween the TEM cell and the hybrid coupler on the measured 
data is analyzed and the methodology for correcting the data is proposed. The proposed methodology is validated on the measurements of the small loop, which is a structure with the known radiation characteristics. Furthermore, multiple dipole moments are extracted from the measurements of two synchronous buck converters presented in [16]. The converters are used as the examples of complex unintentional radiators. The measurements are performed by positioning the converters in different orientations relative to the septum of the TEM cell by using the modular design with two card-edge connectors proposed in [17]. Finally, the radiated fields of the converters are calculated from the extracted dipole moments.

This paper is structured as follows. Section II gives the equations for calculation of radiated fields. Section III describes the measurement setup and presents the methodology for correcting the effects of the impedance mismatch between the TEM cell and the hybrid coupler. Section IV validates the proposed methodology by applying it to the measurements of a small loop. Section V presents the calculation of the radiated fields of two synchronous buck converters. Section VI concludes the paper.

\section{THEORY}

\section{A. Coupling of a Device Under Test and a TEM cell}

A device under test (DUT) is placed centrally $(x=0)$ inside a TEM cell (Fig. 1). The DUT is mounted on the PCB that covers the TEM cell opening [18]. Operation of the DUT excites the TEM mode of the TEM cell. The forward $\left(\mathbf{a}_{\mathbf{0}}=\left|\mathbf{a}_{\mathbf{0}}\right| \cdot \mathrm{e}^{\jmath \cdot \psi_{a_{0}}}\right)$ and backward $\left(\mathbf{b}_{\mathbf{0}}=\left|\mathbf{b}_{\mathbf{0}}\right| \cdot \mathrm{e}^{\jmath \cdot \psi_{b_{0}}}\right)$ excitation coefficients of the TEM mode are given by [2]:

$\left(\begin{array}{l}\mathrm{a} 0 \\ \mathrm{~b}_{0}\end{array}\right)=-0.5 \cdot\left(\mathrm{p}_{\mathrm{y}} \mp \jmath \cdot \mathrm{k}_{0} \cdot \mathrm{m}_{\mathrm{x}}\right) \cdot \mathrm{e}_{\mathrm{oy}}$,

where $\mathbf{p}_{\mathbf{y}}=\left|\mathbf{p}_{\mathbf{y}}\right| \cdot \mathrm{e}^{\jmath \cdot \psi^{p}} y$ and $\mathbf{m}_{\mathbf{x}}=\left|\mathbf{m}_{\mathbf{x}}\right| \cdot \mathrm{e}^{\jmath \cdot \psi} \boldsymbol{m} \boldsymbol{x}$ are the electric and magnetic dipole moments, respectively, $k_{0}$ is the

wavenumber and $e_{0 y} y$ is the electric field component of the normalized TEM mode in the TEM cell at $x=0$ calculated by [19]:

$$
\begin{aligned}
\mathrm{e}_{0 \mathrm{y}}= & \frac{2}{\mathrm{w}} \cdot \sqrt{\mathrm{Z}_{\mathrm{c}}} \cdot \sum_{\mathrm{m}=1,3,5, \ldots}^{\infty} \frac{\cosh [\mathrm{M} \cdot(\mathrm{h}-\mathrm{y})]}{\sinh (\mathrm{M} \cdot \mathrm{h})} \\
& \cos (\mathrm{M} \cdot \mathrm{x}) \cdot \sin (\mathrm{M} \cdot \mathrm{w}) \cdot \mathrm{J}_{0}(\mathrm{M} \cdot \mathrm{g})
\end{aligned}
$$

where $M=m \cdot \pi /(2 \cdot w), Z_{c}$ is the characteristic impedance of the TEM cell $\left(Z_{c}=50 \Omega\right), J_{n}(x)$ is the Bessel function of the first kind, $2 \cdot w, 2 \cdot h, g$ and $y$ are the TEM cell width, the height, the gap width and the distance of the DUT above the TEM cell septum, respectively, as shown in Fig. 1(b). In this paper, complex quantities are denoted by $\mathbf{X}=|\mathbf{X}| \cdot \mathrm{e}^{\jmath \cdot \psi X}$, where $|\mathbf{X}|$ is the magnitude of $\mathbf{X}$, while $\psi_{X}$ is its phase.

The contribution of the electric and magnetic dipole mo-ments can be separated by using the phase information of the excitation coefficients given by (1). The sum of the excitation coefficients corresponds to the contribution of the electric dipole moment (and it is equal to twice the common-mode coupling), while the difference corresponds to the contribu-tion of the magnetic dipole moment (and it is equal to the differential-mode coupling):

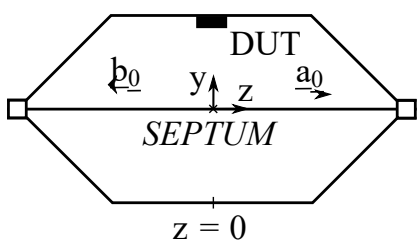

(a)

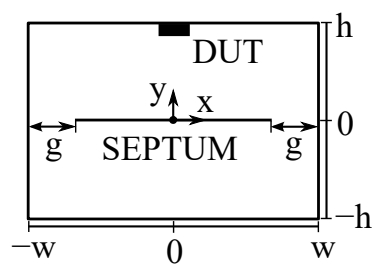

(b)
Fig. 1. DUT inside a TEM cell: (a) yz cross-section and (b) xy cross-section.

$$
\begin{aligned}
& \mathrm{a}_{0}+\mathrm{b}_{0}=-\mathrm{p}_{\mathrm{y}} \cdot \mathrm{e}_{\mathrm{oy}}, \\
& \mathrm{a}_{0}-\mathrm{b}_{0}=-\jmath \cdot \mathrm{k}_{0} \cdot \mathrm{m}_{\mathrm{x}} \cdot \mathrm{e}_{\mathrm{oy}} .
\end{aligned}
$$

\section{B. Calculation of Radiated Fields from Dipole Moments}

The DUT generates the fields in all directions. However, only the component of the electric dipole moment of the DUT which is aligned to the $\mathrm{y}$ axis of the TEM cell and only the component of the magnetic dipole moment of the DUT which is aligned to the $x$ axis of the TEM cell excite the TEM mode of the TEM cell. This allows to extract multiple dipole moments by rotating the DUT inside the TEM cell [2]-[4]. It is useful to express the dipole moments in terms of the coordinate system of the DUT, while retaining the coordinate system of the TEM cell. Therefore, two coordinate systems are assigned, unprimed to the TEM cell

$(\mathrm{x}, \mathrm{y}, \mathrm{z})$ and primed to the DUT $\left(\mathrm{x}^{\prime}, \mathrm{y}^{\prime}, \mathrm{z}^{\prime}\right)$.

Three orthogonal electric and three orthogonal magnetic dipole moments can be extracted by positioning the DUT inside the TEM cell in three orthogonal positions relative to the septum of the TEM cell. The radiated fields of the DUT can be calculated by combining the contributions of each of the extracted dipole moments as (expressed in the rectangular coordinates of the DUT) [2]:

$$
\begin{aligned}
& \mathbf{E}_{\mathbf{x}^{\prime}, \mathbf{m}}=\mathbf{K}_{\mathbf{E}} \cdot \frac{\mathcal{l}}{r^{2}} \cdot k_{0} \cdot\left(z^{\prime} \cdot \mathbf{m}_{\mathbf{y}^{\prime}}-y^{\prime} \cdot \mathbf{m}_{\mathbf{z}^{\prime}}\right), \\
& \mathbf{E}_{\mathbf{x}^{\prime}, \mathbf{p}}=-\mathbf{K}_{\mathbf{E}} \cdot \frac{1}{r^{3}} \cdot\left[-\left(y^{\prime 2}+z^{\prime 2}\right) \cdot \mathbf{p}_{\mathbf{x}^{\prime}}\right. \\
& \left.+x^{\prime} \cdot y^{\prime} \cdot r^{3} \mathbf{p}_{\mathbf{y}^{\prime}}+z^{\prime} \cdot x^{\prime} \cdot \mathbf{p}_{\mathbf{z}^{\prime}}\right] \text {, } \\
& \mathbf{E}_{\mathbf{y}^{\prime}, \mathbf{m}}=\mathbf{K}_{\mathbf{E}} \cdot \frac{\mathcal{l}}{r^{2}} \cdot k_{0} \cdot\left(-z^{\prime} \cdot \mathbf{m}_{\mathbf{x}^{\prime}}+x^{\prime} \cdot \mathbf{m}_{\mathbf{z}^{\prime}}\right) \text {, } \\
& \mathbf{E}_{\mathbf{y}^{\prime}, \mathbf{p}}=-\mathbf{K}_{\mathbf{E}} \cdot \frac{1}{r^{3}} \cdot\left[-\left(z^{\prime 2}+x^{\prime 2}\right) \cdot \mathbf{p}_{\mathbf{y}^{\prime}}\right. \\
& \left.+x^{\prime} \cdot y^{\prime} \cdot r^{3} \mathbf{p}_{\mathbf{x}^{\prime}}+y^{\prime} \cdot z^{\prime} \cdot \mathbf{p}_{\mathbf{z}^{\prime}}\right] \text {, } \\
& \mathbf{E}_{\mathbf{z}^{\prime}, \mathbf{m}}=\mathbf{K}_{\mathbf{E}} \cdot \frac{\mathcal{L}}{r^{2}} \cdot k_{0} \cdot\left(y^{\prime} \cdot \mathbf{m}_{\mathbf{x}^{\prime}}-x^{\prime} \cdot \mathbf{m}_{\mathbf{y}^{\prime}}\right), \\
& \mathbf{E}_{\mathbf{z}^{\prime}, \mathbf{p}}=-\mathbf{K}_{\mathbf{E}} \cdot \underline{1} \cdot\left[-\left(x^{\prime 2}+y^{\prime 2}\right) \cdot \mathbf{p}_{\mathbf{z}^{\prime}}\right. \\
& \left.+z^{\prime} \cdot x^{\prime} \cdot \mathbf{p}_{\mathbf{x}^{\prime}}+y^{\prime} \cdot z^{\prime} \cdot \mathbf{p}_{\mathbf{y}^{\prime}}\right], \\
& \mathbf{E}=\left(\mathbf{E}_{\mathbf{x}^{\prime}, \mathbf{m}}+\mathbf{E}_{\mathbf{x}^{\prime}, \mathbf{p}}\right) \cdot \hat{\mathbf{x}}^{\prime}+\left(\mathbf{E}_{\mathbf{y}^{\prime}, \mathbf{m}}+\mathbf{E}_{\mathbf{y}^{\prime}, \mathbf{p}}\right) \cdot \hat{\mathbf{y}^{\prime}} \\
& +\left(\mathbf{E}_{\mathbf{z}^{\prime}, \mathbf{m}}+\mathbf{E}_{\mathbf{z}^{\prime}, \mathbf{p}}\right) \cdot \hat{\mathbf{z}^{\prime}}
\end{aligned}
$$

where $\mathbf{K}_{\mathbf{E}}=-\jmath \cdot k_{0} \cdot \eta_{0} /(4 \cdot \pi), k_{0}=2 \cdot \pi / \lambda, \lambda=c / f$ is the wavelength, $c$ is the speed of light in free space, $f$ is the frequency, while $\eta_{0} \approx 377 \Omega$ is the impedance of free space. 


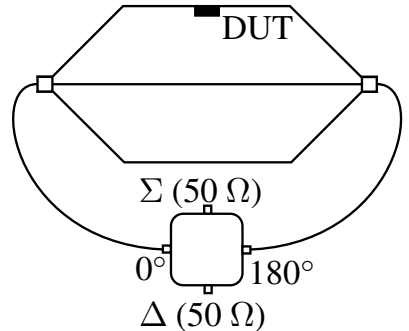

Fig. 2. Schematic representation of the measurement setup. The TEM cell is connected to the ports $0^{\circ}$ and $180^{\circ}$ of the hybrid coupler by two $50-\Omega$ coaxial cables, while the ports $\Sigma$ and $\Delta$ are terminated into $50 \Omega$.

\section{MEASUREMENT SETUP}

\section{A. Description}

A hybrid coupler can be used to generate the sum and difference of the present at the outputs of the TEM cell [3], [4]. The outputs of the

TEM cell are connected to the ports $0^{\circ}$ and $180^{\circ}$ of the hybrid coupler by two coaxial cables, while the ports $\Sigma$ and $\Delta$ of the hybrid coupler are connected to the measuring equipment with the input impedance of $50 \Omega$ or terminated into $50 \Omega$. The schematic representation of the measurement setup is shown in Fig. 2.

Under the assumption that the hybrid coupler generates the ideal sum and difference of the two signals, the voltage $\mathrm{V}_{\mathrm{p}}=\left|\mathrm{V}_{\mathrm{p}}\right| \cdot \mathrm{e}^{J \cdot \psi} \mathrm{p}$ present at the port $\Sigma$ is proportional to the sum, while the voltage $V_{m}$ $=|\mathrm{V} \mathrm{m}| \cdot \mathrm{e}^{\jmath \cdot \psi} \cdot \overrightarrow{\mathrm{Vm}}$ present at the port $\Delta$ of the hybrid coupler is proportional to the difference of the signals present at the outputs of the TEM cell. Considering the normalization used in (2), the voltages are related to the excitation coefficients and to the dipole moments as:

$$
\begin{aligned}
& \mathbf{V}_{\mathbf{p}}=\left(\mathbf{a}_{\mathbf{0}}+\mathbf{b}_{\mathbf{0}}\right) \cdot \sqrt{50}=-\mathbf{p}_{\mathbf{y}} \cdot e_{o y} \cdot \sqrt{50}, \\
& \mathbf{V}_{\mathbf{m}}=\left(\mathbf{a}_{\mathbf{0}}-\mathbf{b}_{\mathbf{0}}\right) \cdot \overline{\sqrt{50}}=-\jmath \cdot k_{0} \cdot \mathbf{m}_{\mathbf{x}} \cdot e_{o y} \cdot \sqrt{50}
\end{aligned}
$$

\section{B. Circuit Model}

The model of the measurement setup is shown in Fig. 3. It consists of a model of the hybrid coupler, a current source, a voltage source, and two transmission line elements. The current source models the contribution of the electric dipole moment (i.e. the common-mode coupling), while the voltage source models the contribution of the magnetic dipole moment (i.e. the differentialmode coupling). The same configuration of sources is used to model the plane wave coupling to multiconductor transmission lines (MTLs) [20]. Each of the two transmission line elements models one half of the TEM cell and one cable. Although a model with more details can be developed (e.g. modelling the TEM cell, cables and transitions separately), the presented model is sufficiently accurate, as shown in Section IV.

The parameters of the circuit model are the characteristic impedance $\mathrm{Z}_{0}$ of the transmission lines (typically equal to $50 \Omega$ ) and the frequency $\mathrm{f} E=\pi / 2$ at which their electrical lengths are equal to $\pi / 2$.

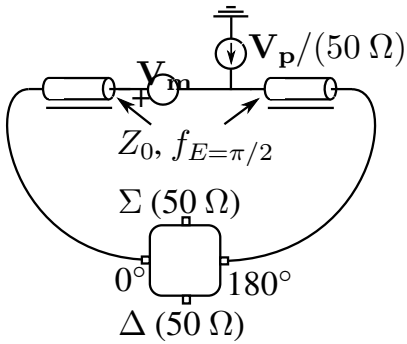

Fig. 3. Model of the measurement setup.

\section{Impedance Mismatch}

The TEM cell and the hybrid coupler are mismatched which has an impact on the generation of the sum and difference of the signals e present at the outputs of the TEM cell. The impedance mismatch can be illustrated by analyzing the input impedances of the ports $\Sigma$ and $\Delta$ of the hybrid coupler when no DUT is present inside the TEM cell $\left(\mathrm{V}_{\mathrm{p}}=\mathrm{V}_{\mathrm{m}}=0 \mathrm{~V}\right.$ in Fig. 3$)$ and under the assumption that all elements of the measurement setup are ideal.

When a signal is applied to the port $\Sigma$ of the hybrid coupler, an ideal common-mode signal appears at its ports $0^{\circ}$ and $180^{\circ}$. For the common-mode signal, the measurement setup can be divided in two at the point of symmetry, with that point left open. The point of symmetry is the center of the TEM cell, $z=0$ in Fig. 1(a), i.e. it is the point between the two transmission line elements in Fig. 3. The input impedance of the port $\Sigma$ can be described by the equation for the impedance of the transmission line terminated into an open circuit [21]:

$\mathbf{Z}_{\mathbf{i n}, \boldsymbol{\Sigma}}=-\jmath \cdot Z_{0} \cdot \cot (E)$,

where $\mathrm{Z}_{0}$ and $\mathrm{E}$ are the characteristic impedance and the equivalent electrical length of one half of the measurement setup (one half of the signal path through the hybrid cou-pler, one cable and one half of the TEM cell), respectively, $E=(2 \cdot \pi \cdot \mathrm{f} / \mathrm{v}) \cdot 1, \mathrm{f}$ is the frequency, $\mathrm{v}$ is the speed of propagation of an electromagnetic wave through the measure-ment setup, while 1 is the physical length of one half of the measurement setup.

Equivalently, when a signal is applied to the port $\Delta$ of the hybrid coupler, an ideal differential-mode signal appears at its ports $0^{\circ}$ and $180^{\circ}$. The point of symmetry can be grounded and the input impedance of the port $\Delta$ can be described by the equation for the impedance of the transmission line terminated into a short circuit [21]:

$\mathbf{Z}_{\mathbf{i n}, \boldsymbol{\Delta}}=\jmath \cdot Z_{0} \cdot \tan (E)$.

The equations (14) and (15) show that the input impedances of the ports $\Sigma$ and $\Delta$ of the hybrid coupler when the TEM cell is connected to the ports $0^{\circ}$ and $180^{\circ}$ are frequency dependent, i.e. the TEM cell and the hybrid coupler are mismatched, even if all elements of the measurement setup are ideal. The mismatch is a consequence of the phase shift which is related to the propagation of the signal through the measurement setup. This is the dominant source of the impedance mismatch. Additionally, the elements of the measurement setup are not 
ideal, i.e. their input impedances are not exactly $50 \Omega$, which also contributes to the impedance mismatch.

\section{Transfer Functions of the Measurement Setup}

The impact of the measurement setup can be described by a set of transfer functions as follows. The voltages present at the ports $\Sigma\left(\mathrm{V}_{\Sigma}=\right.$ $\left.\left|\mathrm{V}_{\Sigma}\right| \cdot \mathrm{e}^{J \cdot \psi \Sigma}\right)$ and $\Delta\left(\mathrm{V}_{\Delta}=\left|\mathrm{V}_{\Delta}\right| \cdot \mathrm{e}^{J \cdot \psi \Delta}\right)$ of the hybrid coupler are related to $V_{p}$ and $V_{m}$ as:

$$
\begin{aligned}
\mathbf{V}_{\boldsymbol{\Sigma}} & =\mathbf{V}_{\mathbf{p}} \cdot \mathbf{H}_{\mathbf{c c}}+\mathbf{V}_{\mathbf{m}} \cdot \mathbf{H}_{\mathbf{c d}} \\
\mathbf{V}_{\boldsymbol{\Delta}} & =\mathbf{V}_{\mathbf{m}} \cdot \mathbf{H}_{\mathbf{d d}}+\mathbf{V}_{\mathbf{p}} \cdot \mathbf{H}_{\mathbf{d c}}
\end{aligned}
$$

where $\mathrm{H}_{\mathrm{cc}}=\left|\mathrm{H}_{\mathrm{cc}}\right| \cdot \mathrm{e}^{J} \cdot \psi_{\mathrm{cc}}, \mathrm{H}_{\mathrm{dc}}=\left|\mathrm{H}_{\mathrm{dc}}\right| \cdot \mathrm{e}^{J \cdot \psi \mathrm{dc}}, \mathrm{H}_{\mathrm{cd}}=\left|\mathrm{H}_{\mathrm{cd}}\right| \cdot \mathrm{e}^{J}$ $\psi \mathrm{cd}$ and $\mathrm{H}_{\mathrm{dd}}=\left|\mathrm{H}_{\mathrm{dd}}\right| \cdot \mathrm{e}^{\jmath} \psi \psi \mathrm{dd}$ are the common-to-common, commonto-differential, differential-to-common and differential-to-differential transfer functions of the measure-ment setup, respectively. The transfer functions can be de-termined from the circuit model of the measurement setup (Fig. 3).

The model is generated in two steps. Firstly, the hybrid coupler is characterized. In this paper, the hybrid coupler PH030 with the operating frequency range from $5 \mathrm{MHz}$ to $1000 \mathrm{MHz}$ [22] is measured by a 2-port vector network analyzer (VNA). While one set of measurements is performed, another two ports of the hybrid coupler are terminated into $50 \Omega$. The measured matrices are combined into a 4-port matrix.

Secondly, the parameters of the model (Fig. 3) are adjusted to fit the simulated input impedances of the hybrid coupler $Z_{\text {in, }, \Sigma}$ and $Z_{\text {in, }, \Delta}$ to those calculated from the measured $\mathrm{S}$ parameters. The measurements are performed by a VNA, with its port 1 connected to the port $\Sigma$ and its port 2 connected to the port $\Delta$ of the hybrid coupler, while the ports $0^{\circ}$ and $180^{\circ}$ of the hybrid coupler are connected to the TEM cell by the coaxial cables.

Once the model is built, the transfer functions are simulated as follows. The signals that are generated at the ports $\Sigma$ and $\Delta$ which are terminated into $50 \Omega$ when the model is excited by the unity common-mode signal $\left(\mathrm{V}_{\mathrm{p}}=1 \mathrm{~V}, \mathrm{~V}_{\mathrm{m}}=0 \mathrm{~V}\right.$ in Fig. 3) correspond to $\mathrm{H}_{\mathrm{cc}}$ and $\mathrm{H}_{\mathrm{dc}}$, respectively. Equivalently, the signals that are generated at the ports $\Sigma$ and $\Delta$ which are terminated into $50 \Omega$ when the model is excited by the unity differential-mode signal $\left(\mathrm{V}_{\mathrm{p}}=0 \mathrm{~V}\right.$, $\mathrm{V}_{\mathrm{m}}=1 \mathrm{~V}$ in Fig. 3) correspond to $\mathrm{H}_{\mathrm{cd}}$ and $\mathrm{H}_{\mathrm{dd}}$, respectively.

\section{E. Correcting the Measured Data}

Re-arranging (16) and (17) allows to calculate the voltages $V_{p}$ and $\mathrm{V}_{\mathrm{m}}$ from the voltages $\mathrm{V}_{\Sigma}$ and $\mathrm{V}_{\Delta}$ measured at the ports of the hybrid coupler and from the known transfer functions of the measurement setup:

$$
\begin{aligned}
& \mathbf{V}_{\mathbf{p}}=\frac{\left|\mathbf{V}_{\underline{\Sigma}}\right| \cdot \underline{\mathrm{e}}^{\gamma \cdot \psi_{\underline{\Sigma}}} \cdot \underline{\mathbf{H}}_{\mathbf{d d}}=\left|\mathbf{V}_{\underline{\Delta}}\right| \cdot \mathrm{e}^{\jmath \cdot \psi_{\Delta}} \cdot \underline{\mathbf{H}}_{\mathbf{c d}}}{\mathbf{H}_{\mathbf{c c}} \cdot \mathbf{H}_{\mathbf{d d}}-\mathbf{H}_{\mathbf{c d}} \cdot \mathbf{H}_{\mathbf{d c}}}, \\
& \mathbf{V}_{\mathbf{m}}=\frac{\left|\mathbf{V}_{\underline{\Delta}}\right| \cdot-\underline{\mathrm{e}}^{\jmath \cdot \psi_{\underline{\Delta}}} \cdot \underline{\mathbf{H}}_{\mathbf{c c}}=\left|\mathbf{V}_{\underline{\Sigma}}\right| \cdot \cdot \underline{\mathrm{e}}^{\jmath \cdot \psi_{\underline{\Sigma}}} \cdot \underline{\mathbf{H}}_{\mathbf{d c}}}{\mathbf{H}_{\mathbf{c c}} \cdot \mathbf{H}_{\mathbf{d d}}-\mathbf{H}_{\mathbf{c d}} \cdot \mathbf{H}_{\mathbf{d c}}} .
\end{aligned}
$$

The voltages $\mathrm{V}_{\Sigma}$ and $\mathrm{V}_{\Delta}$ are typically measured by a spectrum analyzer (SA), i.e. only the magnitude is measured.

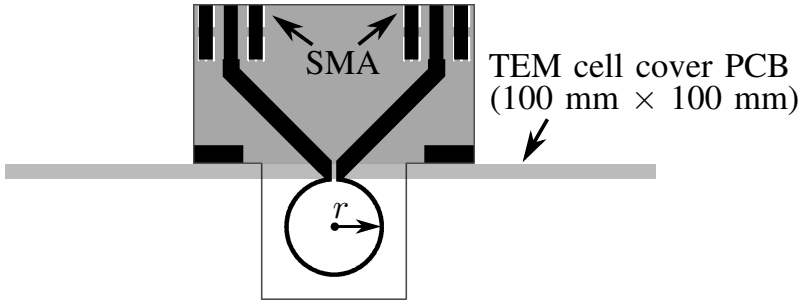

(a)

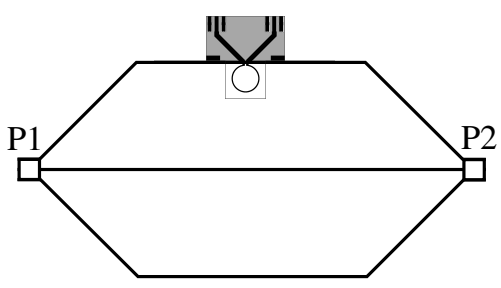

(b)

Fig. 4. Validation structure: (a) the small loop soldered to a $100 \mathrm{~mm} \times 100 \mathrm{~mm}$ PCB at a $90^{\circ}$ angle which is used as a cover for the TEM cell opening (b) orientation of the loop inside the TEM cell.

Therefore, instead of the exact solution, only the solution for $\psi \Sigma=\psi \Delta=0^{\circ}$ can be calculated. However, if $\mathrm{H}_{\mathrm{cc}}$ and $\mathrm{H}_{\mathrm{dd}}$ are much larger in magnitude than $\mathrm{H}_{\mathrm{dc}}$ and $\mathrm{H}_{\mathrm{cd}}$, which is typically the case, the impact of the phase information of the measured voltages can be neglected. The impact of the phase information is further analyzed in Section IV.

Once the voltages $\mathrm{V}_{\mathrm{p}}$ and $\mathrm{Vm}_{\mathrm{m}}$ are determined, the elec-tric and

magnetic dipole moments can be calculated by (12) and (13). The proposed methodology is repeated for multiple orientations of the DUT inside the TEM cell to extract the electric and magnetic dipole moments of the DUT. Finally, the radiated fields are calculated by (5)-(11). Since the phases of the extracted dipole moments are unknown if the coupling is measured by the SA, the radiation digram can be calculated by

setting $\psi_{\mathrm{m}_{\mathrm{X}}}=\psi \mathrm{m}_{\mathrm{y}}{ }^{\prime}=\psi \mathrm{m}_{\mathrm{z}^{\prime}}=0^{\circ}$ and $\psi \mathrm{p}_{\mathrm{x}}{ }^{\prime}=\psi \mathrm{p}_{\mathrm{y}}{ }^{\prime}=\psi \mathrm{p}_{\mathrm{z}}{ }^{\prime}=90^{\circ}$. For those values, the contributions of all dipole moments are in-phase and the calculated radiated fields represent the worst-case scenario.

\section{VALIDATION}

\section{A. Measurements of a Small Loop}

A small loop is measured to validate the proposed method-ology. The measured structure is shown in Fig. 4(a). It consists of a small loop, two $50-\Omega$ feed lines and two end-launch subminiature version $A$ (SMA) connectors. The loop has a radius of $3.5 \mathrm{~mm}$ and it is small compared to the wavelength in the frequency range of interest (up to $1000 \mathrm{MHz}$ ). The structure is soldered to a $100 \mathrm{~mm} \times 100 \mathrm{~mm}$ PCB at a $90^{\circ}$ angle. The PCB is used as a cover for the TEM cell opening. It allows to position the loop inside, while keeping the feed lines outside of the TEM cell.

The coupling of the loop and the TEM cell is measured by the measurement setup described in Section III. The orientation of the loop in the TEM cell is shown in Fig. 4(b). The port 1 of the VNA is connected to one of the two SMA connectors of the loop 4 (the second connector is terminated 
TABLE I

RESUltS OF THE FSV TECHNIQUE APPLIED TO THE MAGNITUDES OF THE MEASURED AND MODELLED INPUT IMPEDANCES OF THE HYBRID COUPLER.

\begin{tabular}{ccc} 
FSV measure & $\left|\mathbf{Z}_{\text {in }, \boldsymbol{\Sigma}}\right|$ & $\left|\mathbf{Z}_{\mathbf{i n}, \boldsymbol{\Delta}}\right|$ \\
\hline $\begin{array}{c}\text { Global difference measure } \\
(\text { GDM), weighted }\end{array}$ & 0.313 (good) & 0.142 (very good) \\
\hline GDM Grade (threshold $=85 \%)$ & 4 & 3 \\
\hline GDM Spread (threshold $=85 \%)$ & 4 & 3 \\
\hline
\end{tabular}

into $50 \Omega$ ), while its port 2 is connected to the hybrid coupler. Two measurements are performed, in the first one the VNA is connected to the port $\Sigma$ of the hybrid coupler, while in the second one it is connected to the port $\Delta$ of the hybrid coupler. The other port of the hybrid coupler $(\Sigma$ or $\Delta)$ is terminated into $50 \Omega$.

The coupling of the small loop to the TEM cell is also measured by connecting the port 2 of the VNA directly to the outputs of the TEM cell (P1 and P2 in Fig. 4(b)). Two measurements are performed, one for each output. While the VNA is connected to one of the outputs, the other output is terminated into $50 \Omega$. Since the data measured by the VNA contains the phase information, the operation of the hybrid coupler is mimicked by performing the sum and the difference of the two measurements. The sum corresponds to the coupling measured at the port $\Sigma$, while the difference corresponds to the coupling measured at the port $\Delta$ of the hybrid coupler. The sum and the difference are denoted as the reference results and they are used to validate the proposed methodology.

The coupling measured by the two aforementioned methods and the error expressed relative to the reference results are shown in Fig. 5. The ringing in the data measured by the TEM cell and the hybrid coupler, which is the consequence of the impedance mismatch between them, can be observed. Similar ringing can be observed in the measurements presented in [14], [15]. The maximum absolute value of the error for the measurements of the coupling which corresponds to the electric dipole moment is $5.9 \mathrm{~dB}$, while that which corresponds to the magnetic dipole moment is $8.5 \mathrm{~dB}$.

\section{B. Application of the Proposed Methodology}

The model of the measurement setup (Fig. 3) is fitted to the measurements and the obtained model parameters are $\mathrm{Z} 0=50 \Omega$ and $\mathrm{fE}=\pi / 2=49.2 \mathrm{MHz}$. The comparison of the measured and modelled input impedances of the ports $\Sigma$ and $\Delta$ of the hybrid coupler is shown in Fig. 6. The modelled input impedance of the port $\Sigma$ shows a good agreement, while that of the port $\Delta$ shows a very good agreement to the measurements in the frequency range from $30 \mathrm{MHz}$ to $1000 \mathrm{MHz}$ (the frequency range of interest for the radiated emissions [1]) according to the feature selective validation (FSV) technique [23][25]. The results of the FSV technique are summarized in Table I.

The input impedances show a strong dependence on the frequency, which confirms the theoretical analysis of the impedance mismatch between the TEM cell and the hybrid

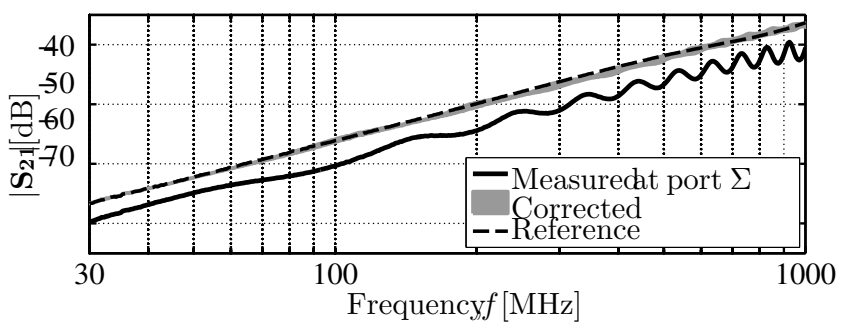

(a)

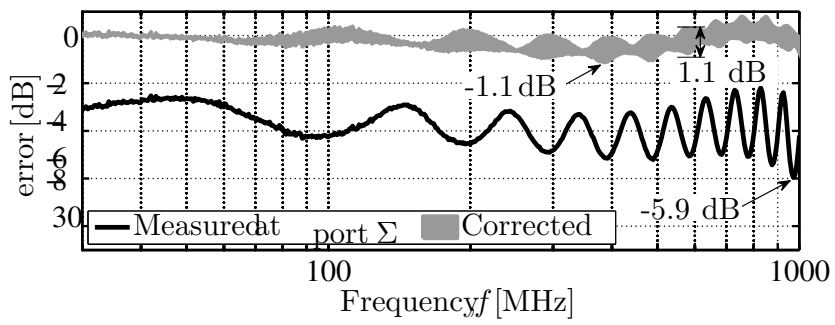

(b)

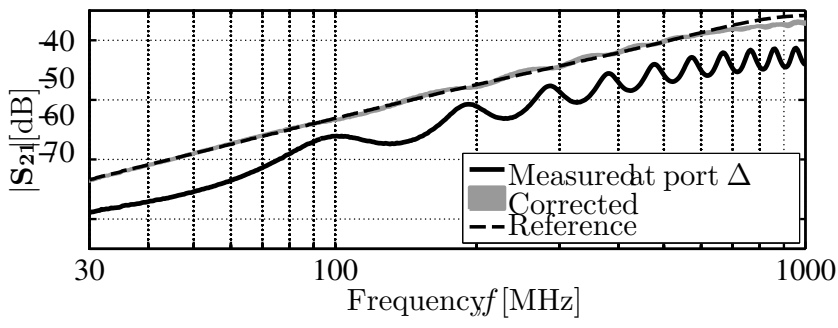

(c)

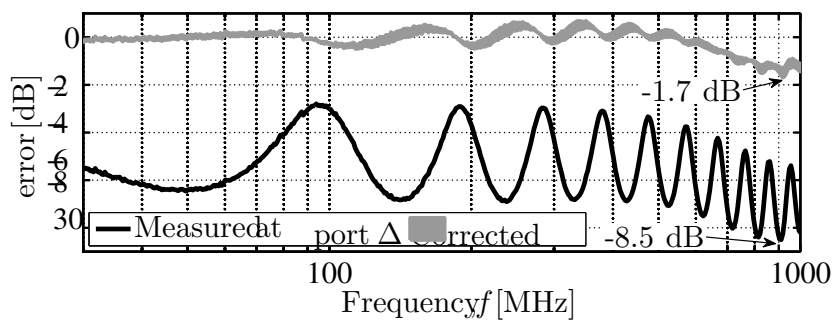

(d)

Fig. 5. Measurements of the small loop: (a) coupling which corresponds to the electric dipole moment, (b) the error expressed relative to the reference results, (c) coupling which corresponds to the magnetic dipole moment, and (d) the error expressed relative to the reference results.

coupler. Furthermore, the input impedances of the ports $\Sigma$ and $\Delta$ of the hybrid coupler when its ports $0^{\circ}$ and $180^{\circ}$ are terminated into $50 \Omega$ are added to Fig. 6 (denoted by "Reference"). Those impedances are not exactly $50 \Omega$ since the hybrid coupler is not ideal. More specifically, they vary from $35 \Omega$ to $53 \Omega$ in the frequency range from $30 \mathrm{MHz}$ to $1000 \mathrm{MHz}$. However, this variation is significantly smaller than the one observed in the case when the TEM cell is connected to the ports $0^{\circ}$ and $180^{\circ}$ of the hybrid coupler. This additionally confirms that the phase shift which is related to the propagation of the signal through the measurement setup is the dominant source of the impedance mismatch.

The simulated transfer functions of the measurement setup are shown in Fig. 7. The impact of the impedance mismatch is seen as the ringing of the transfer functions. The equations 


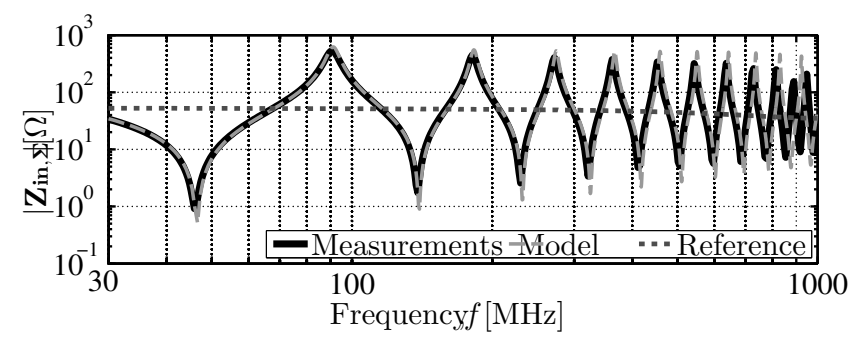

(a)

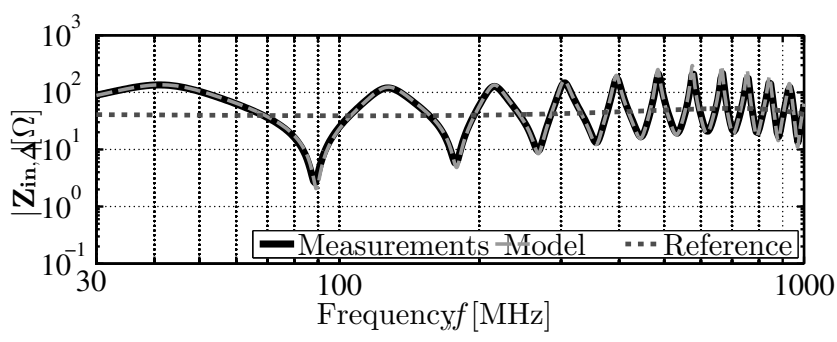

(b)

Fig. 6. Comparison of the magnitudes of the measured and modelled input impedances of the hybrid coupler: (a) $\mathbf{Z}_{\mathbf{i n}, \boldsymbol{\Sigma}}$ and (b) $\mathbf{Z}_{\mathbf{i n}, \boldsymbol{\Delta}}$. The measured impedances when the ports $0^{\circ}$ and $180^{\circ}$ of the hybrid coupler are terminated into $50 \Omega$ are denoted by "Reference".

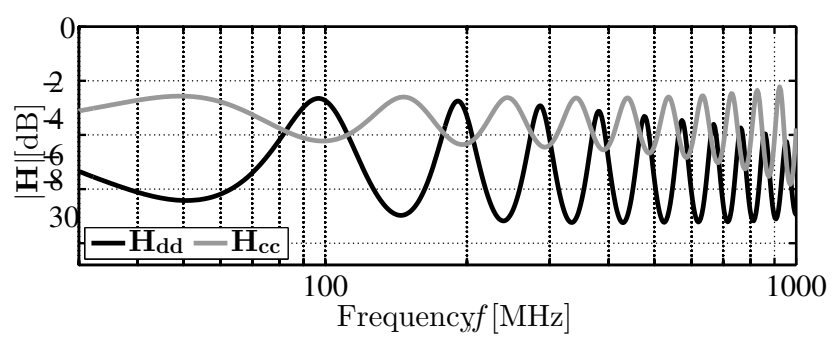

(a)

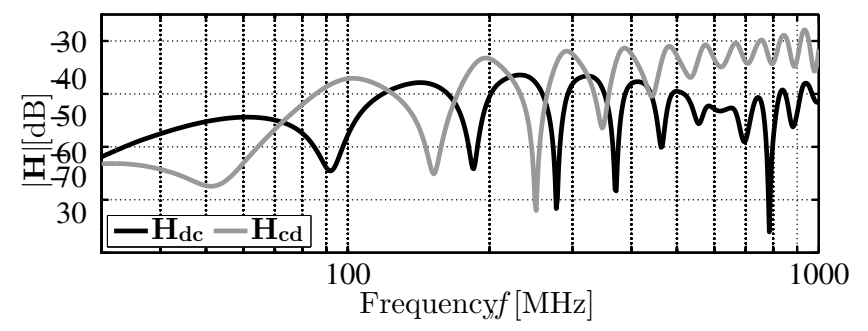

(b)

Fig. 7. Magnitudes of the simulated transfer functions: (a) $\mathbf{H}_{\mathbf{d d}}$ and $\mathbf{H}_{\mathbf{c c}}$, and (b) $\mathbf{H}_{\mathbf{d c}}$ and $\mathbf{H}_{\mathbf{c d}}$.

(18) and (19) are applied to the measured S parameters (V is replaced by $\mathrm{S}_{21}$ in the equations). The corrected results and the error expressed relative to the reference measurements are shown in Fig. 5. Although the phases $\psi \Sigma$ and $\psi \Delta$ are known in this case as the measurements are performed by the VNA, they are swept from $0^{\circ}$ to $360^{\circ}$ with the step of $1^{\circ}$ to analyze their impact. The corrected results in Fig. 5 show all the data obtained by sweeping the phases.

After the proposed methodology is applied, the error is significantly reduced, which validates the proposed methodology.

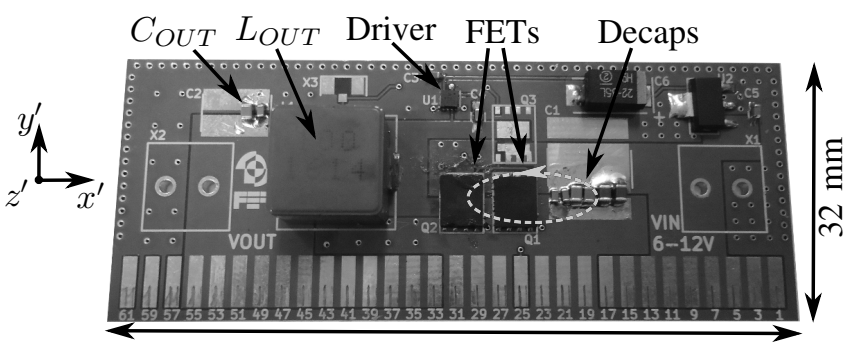

$81 \mathrm{~mm}$

(a)

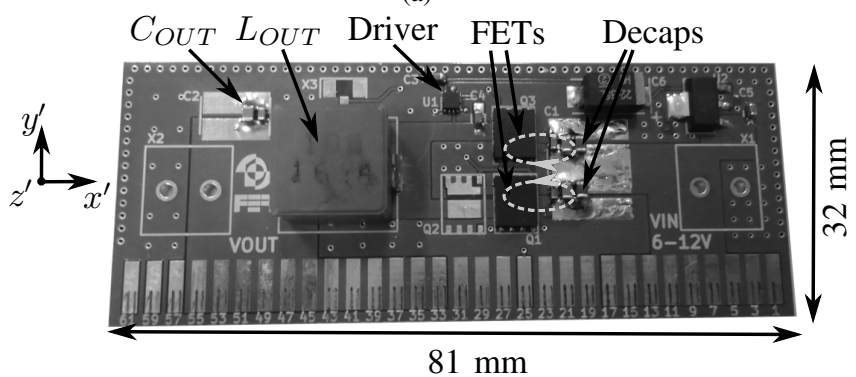

(b)

Fig. 8. Two converters [16]: (a) with one and (b) with two loops in the input decoupling network. The loops are indicated by the dashed gray circles.

The maximum absolute error for the measurements of the coupling which corresponds to the electric dipole moment is 1.1 $\mathrm{dB}$, while that which corresponds to the magnetic dipole moment is $1.7 \mathrm{~dB}$. The uncertainty due to the phase informa-tion (the difference between the maximum and the minimum value obtained at the same frequency while sweeping the phases) is smaller than $1.1 \mathrm{~dB}$ in the complete frequency range of interest.

\section{RESULTS}

\section{A. Measurements of Synchronous Buck Converters}

The proposed methodology is applied to the measured data of two synchronous buck converters presented in [16]. The converters are used as the examples of complex unintentional radiators. The converters are briefly described as follows.

The converters operate at the switching frequency of $300 \mathrm{kHz}$. The input voltage of $12 \mathrm{~V}$ is converted into the output voltage of $5 \mathrm{~V}$ with the output current of $3 \mathrm{~A}$. The converters are designed as nonregulated, i.e. the control signal (the pulse width modulation, PWM signal) is generated by the signal generator. The first converter is designed with one resonant loop. The input decoupling network of the second converter is designed in such a way that forces the resonant current to divide into two loops in which it flows in opposite directions, and which reduces the radiated fields. Each converter is designed on a 1.55-mm thick 2-layer PCB. The dimensions of the PCBs are $81 \mathrm{~mm} \times 32 \mathrm{~mm}$. All components are placed on the top layer. The bottom layer is a solid ground plane. The top views of the converters are shown in Fig. 8.

To position the converters inside the TEM cell, the mod-ular design presented in [17] is used. It consists of two $100 \mathrm{~mm} \times 100 \mathrm{~mm}$ PCBs which are used as the covers for the TEM cell opening. The first cover PCB contains the straight, while the second contains the angled (90) 62-pin card-edge connector. Both cover PCBs contain two terminal blocks which provide the input voltage and the load and one 


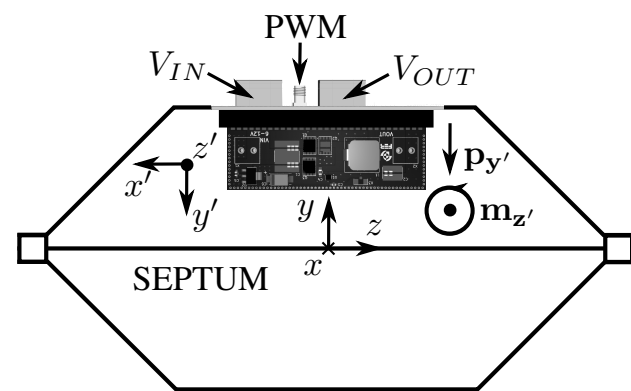

(a)

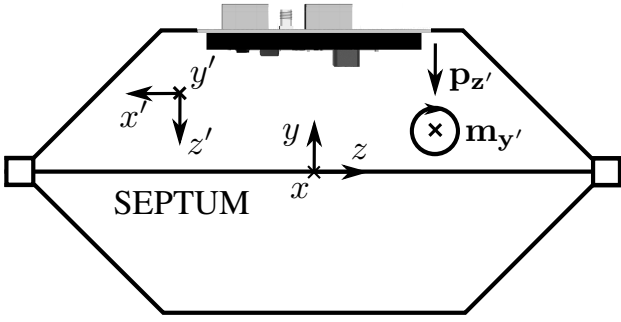

(b)

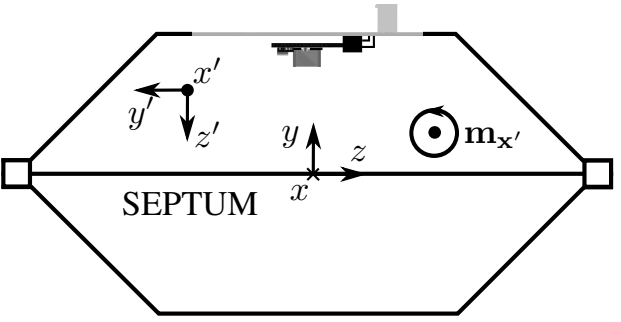

(c)

Fig. 9. Orientations of the converter inside the TEM cell. The coupled voltages correspond to the dipole moments of the measured converter: (a) $\mathrm{m}_{\mathrm{z}^{\prime}}$ and $\mathrm{p}_{\mathrm{y}^{\prime}}$, (b) $\mathrm{m}_{\mathrm{y}^{\prime}}$ and $\mathrm{p}_{\mathrm{z}^{\prime}}$ a) $\mathrm{m}_{\mathrm{x}^{\prime}}$.

MA connector which provides the control signal for the converters. The cover PCBs allow to position the converters inside the TEM cell in different orientations relative to the septum and to provide the required signals to the converters.

The orientations of the converters inside the TEM cell and the corresponding dipole moments are shown in Fig. 9. Five dipole moments are extracted for the measured converters. To extract the sixth dipole moment, the converters should be rotated by $90^{\circ}$ about the $\mathrm{x}$ axis relative to the orientation shown in Fig. 9(a). This is not possible for the measured converters since their lengths $(81 \mathrm{~mm})$ are larger than the distance from the septum of the TEM cell to its top wall (44 mm).

The coupled voltages measured by the SA and presented in [16] are corrected by the proposed methodology. The dipole moments are extracted from the corrected data by (18) and (19) (for $\psi \Sigma=\psi \Delta=0 \circ$ ) and their magnitudes are shown in Fig. 10.

The worst-case radiated fields $(\psi \mathrm{mx}=\psi \mathrm{my}=\psi \mathrm{mz}=0 \circ$ and $\psi \mathrm{py}=$ $\psi \mathrm{pz}=90^{\circ}$ ) at $3 \mathrm{~m}$ are calculated from five extracted dipole moments by (5)-(11). The maximum values of the magnitudes of the calculated radiated fields as a function of frequency are shown in Fig. 11. The calculated radiated fields of the converter with two loops in the input decoupling network show a 4.3-dB reduction of the value for which the margin to the limit line is the smallest, relative to that of the converter with one loop.

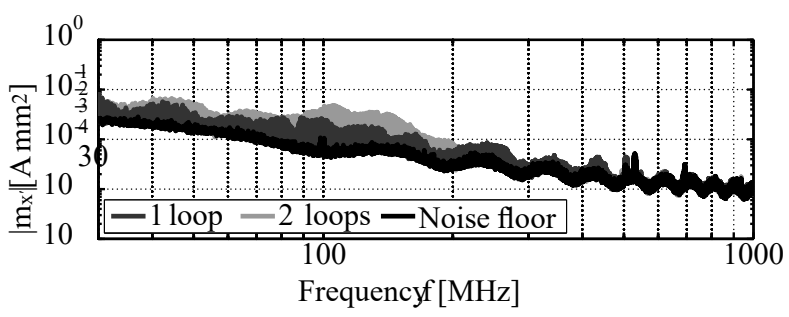

(a)

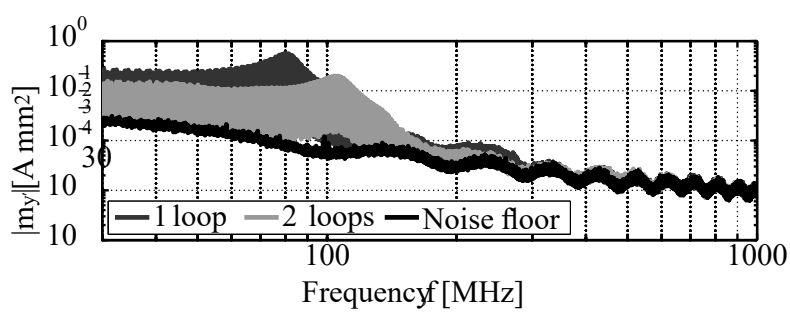

(b)

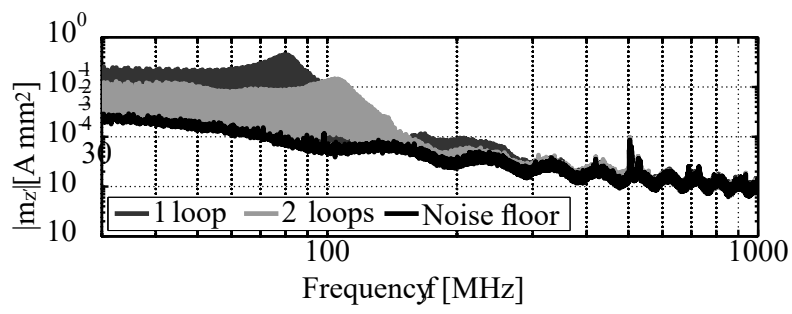

(c)

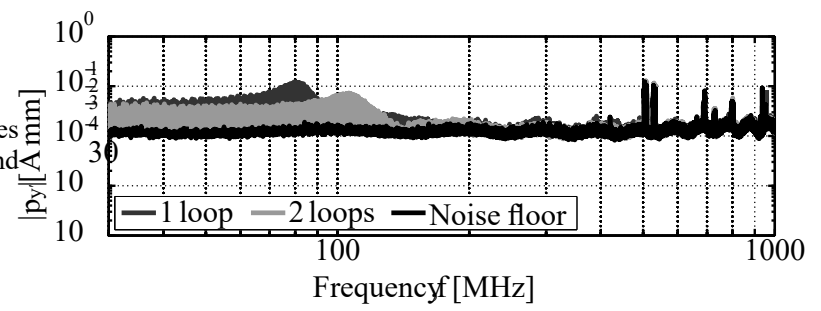

(d)

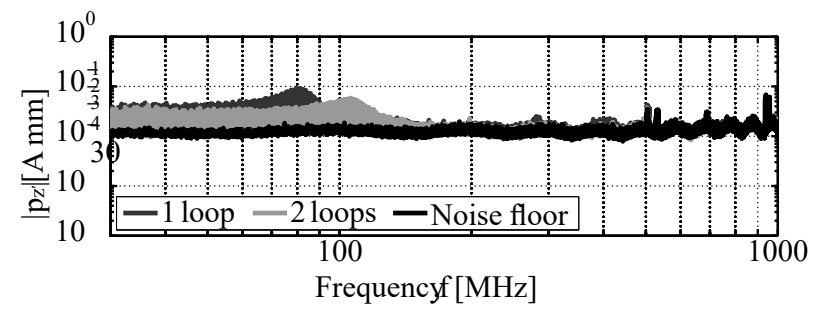

(e)

Fig. 10. The magnitudes of the magnetic dipole moments (a) $\left|\mathrm{m}_{\mathrm{x}^{\prime}}\right|$, (b) $\mid$ my $\mid$, (c) $|\mathrm{mz}|$, and the electric dipole moments (d) $\mid$ py $\mid$ and (e) $|\mathrm{pz}|$ of the two converters

(b)

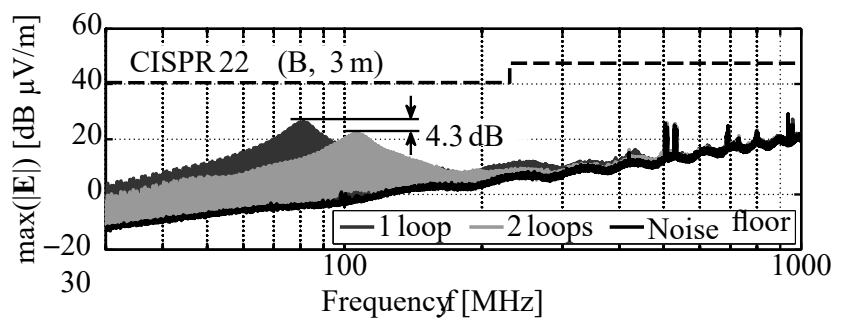

Fig. 11. Maximum values of the magnitudes of the calculated worstcase radiated fields of the two converters, at $3 \mathrm{~m}$. 


\section{B. Discussion}

The dipole moments can help identify the sources ofradiation.

However, they are not sufficient to compare two

unintentional radiators (converters in this paper) as some of the dipole moments may be smaller for one, while others may be smaller for the other radiator. Also, the frequency at which the maximum occurs may not be the same for all dipole moments. The estimated radiated fields allow to perform a direct comparison of the two. Additionally, they allow to compare the radiation to the limit lines specified in the regulations. Although the estimated radiated fields are not validated against the measurements performed in the SAC or at the OATS in this paper, the confidence in their accuracy is based on already published comparisons in [2], [6]-[11].

\section{CONCLUSION}

The impedance mismatch between a TEM cell and a hybrid coupler which is present in the measurement setup used for the measurements of unintentional radiators is analyzed. The mismatch is dominantly a consequence of the phase shift which is related to the propagation of the signal through the measurement setup. The methodology which corrects the impact of the mismatch by modelling the measurement setup by a set of transfer functions is proposed. The proposed methodology is validated on the measurements of the small loop in which the maximum error is reduced from $8.5 \mathrm{~dB}$ to $1.7 \mathrm{~dB}$. Furthermore, the impact of the unknown phase information of the measured data when the coupling is mea-sured by a spectrum analyzer is analyzed and it is shown to be smaller than $1.1 \mathrm{~dB}$ up to 1000 $\mathrm{MHz}$ for the measurement setup used in this paper. The proposed methodology is applied to the measurements of two synchronous buck converters and their worst-case radiated fields are estimated. The estimated radiated fields allow to directly compare the two converters and to compare their radiation to the limit lines specified in the regulations.

\section{REFERENCES}

[1] C. Paul, Introduction to Electromagnetic Compatibility. Wiley, 2006.

[2] P. Wilson, "On correlating TEM cell and OATS emission measurements," IEEE Trans. Electromagn. Compat., vol. 37, no. 1, pp. 116, Feb. 1995.

[3] I. Sreenivasiah, D. C. Chang, and M. T. Ma, "Emission Characteristics of Electrically Small Radiating Sources from Tests Inside a TEM Cell," IEEE Trans. Electromagn. Compat., vol. EMC-23, no. 3, pp. 113-121, Aug. 1981.

[4] G. H. Koepke and M. T. Ma, "A New Method for Determining the Emission Characteristics of an Unknown Interference Source," in IEEE Int. Symp. Electromagn. Compat., Sept. 1982, pp. 1-6.

[5] H. Gerth, S. Fisahn, H. Garbe, and H. Haase, "New Advances on Correlating TEM Cell and OATS Emission Measurements," IEEE Trans. Electromagn. Compat., vol. 52, no. 1, pp. 11-20, Feb. 2010.

[6] A. Nothofer and A. C. Marvin, "Aspects of GTEM to OATS measure-ment correlation," in Int. Conf. Electromagn. Compat., Sept. 1997, pp. 170-174.

[7] R. Guirado, R. del Rio, J. Carpio, F. Garnacho, A. Valladolid, and M. Valcarcel, "Comparison between GTEM and OATS radiated field emission measurements," in IEEE Int. Symp. Electromagn. Compat., Aug. 1995, pp. 338-342.
[8] T. E. Harrington, Z. Chen, and M. D. Foegelle, "GTEM radiated emissions correlation testing and FDTD modeling," in IEEE Int. Symp. Electromagn. Compat., vol. 2, Aug. 1999, pp. 770-775.

[9] S. Pan, J. Kim, S. Kim, J. Park, H. Oh, and J. Fan, "An equivalent three-dipole model for IC radiated emissions based on TEM cell measurements," in IEEE Int. Symp. Electromagn. Compat., July 2010, pp. 652-656.

[10] A. Bhargava, D. Pommerenke, K. Kam, F. Centola, and C. W. Lam, "DC-DC Buck Converter EMI Reduction Using PCB Layout Modification," IEEE Trans. Electromagn. Compat., vol. 53, no. 3, pp. 806-813, Aug. 2011.

[11] K. L. Chua, M. Z. M. Jenu, M. O. Wong, and S. H. Ying, "Radiated emissions estimation of an integrated circuit based on measurements in GTEM cell," in Asia-Pacific Symp. Electromagn. Compat. (APEMC), May 2013, pp. 1-4.

[12] K. Kam, D. Pommerenke, C.-W. Lam, and R. Steinfeld, "EMI Analysis Methods for Synchronous Buck Converter EMI Root Cause Analysis," in Proc. IEEE Int. Symp. Electromagn. Compat., Aug. 2008, pp. 1-7.

[13] S. Deng, T. H. Hubing, and D. G. Beetner, "Using TEM Cell Measure-ments to Estimate the Maximum Radiation From PCBs With Cables Due to Magnetic Field Coupling," IEEE Trans. Electromagn. Compat., vol. 50, no. 2, pp. 419-423, May 2008.

[14] V. Kasturi, S. Deng, T. Hubing, and D. Beetner, "Quantifying electric and magnetic field coupling from integrated circuits with tem cell measurements," in IEEE Int. Symp. Electromagn. Compat., vol. 2, Aug. 2006, pp. 422-425.

[15] T. Mandic, R. Gillon, and A. Baric, "An Experimental Investigation of Four-Port IC-Stripline," in IEEE Int. Workshop on Electromagn. Compat. of Integrated Circuits (EMC Compo), July 2017.

[16] R. Blecic, R. Gillon, B. Nauwelaers, and A. Baric, "EMC-Oriented Design of Output Stage of Synchronous Buck Converter," in IEEE Int. Workshop on Electromagn. Compat. of Integrated Circuits (EMC Compo), July 2017.

[17] R. Blecic, N. Bako, R. Gillon, and A. Baric, "Broadband measurement of the magnetic moment normal to the printed circuit board plane using TEM cell and phase information," in IEEE Int. Symp. Electromagn. Compat. (EMC Europe), Sept. 2013, pp. 955-960.

[18] Integrated Circuits Measurement of Electromagnetic Emissions Part 2: Measurement of Radiated Emissions, TEM Cell and Wideband TEM Cell Method, IEC 61967-2, First Edition Std., 2005.

[19] P. Wilson and M. Ma, "Shielding-Effectiveness Measurements with a Dual TEM Cell," IEEE Trans. Electromagn. Compat., vol. EMC-27, no. 3, pp. 137-142, Aug. 1985.

[20] C. Paul, Analysis of Multiconductor Transmission Lines, 2nd ed. Wiley, 2008.

[21] D. Pozar, Microwave Engineering. Wiley, 2004.

[22] R\&K Company Limited. PH030 datasheet. [Online]. Available: http://www.rk-microwave.com/jp/products/pdf/PH030-0S.pdf

[23] IEEE Recommended Practice for Validation of Computational Electromagnetics Computer Modeling and Simulations. IEEE Std 1597.1, 2008.

[24] A. P. Duffy, A. J. M. Martin, A. Orlandi, G. Antonini, T. M. Benson, and

M. S. Woolfson, "Feature selective validation (FSV) for validation of computational electromagnetics (CEM). part I-the FSV method," IEEE Trans. Electromagn. Compat., vol. 48, no. 3, pp. 449-459, Aug. 2006.

[25] A. Orlandi, A. P. Duffy, B. Archambeault, G. Antonini, D. E. Coleby, and S. Connor, "Feature selective validation (FSV) for validation of computational electromagnetics (CEM). part IIassessment of FSV performance," IEEE Trans. Electromagn. Compat., vol. 48, no. 3, pp. 460-467, Aug. 2006. 


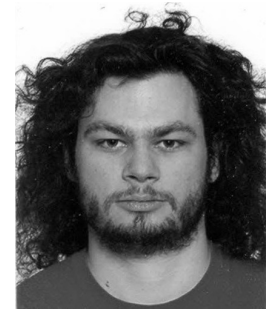

Raul Blecic (S'10) was born in Rijeka, Croatia, in 1986. He received the Dipl.-Ing. degree in electrical engineering from the University of Zagreb, Zagreb, Croatia, in 2009. Currently, he is a double degree $\mathrm{PhD}$ student at the University of Zagreb and $\mathrm{KU}$ Leuven, Belgium. His research interests include electromagnetic compatibility of integrated circuits, integrated circuit design and modelling of microwave structures and components.

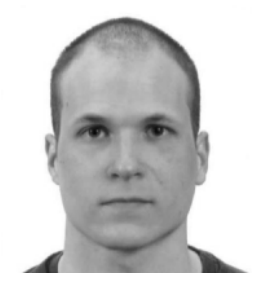

Hrvoje Stimac (S'14) was born in Zagreb, Croatia, in 1988. He received his M.S. degree from the University of Zagreb, Zagreb, Croatia, in 2013. Currently, he is a PhD student at the University of Zagreb. His research is directed towards the design of broadband high common-mode rejection ratio differential probes for voltage and current sensing.

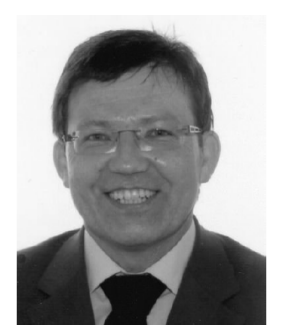

Bart Nauwelaers (S'79-M'88-SM'99) was born in Niel, Belgium on 7 July 1958. He received the M.S. and $\mathrm{Ph}$. D. degrees in electrical engineering from the KU Leuven, Belgium in 1981 and 1988, respectively. He also holds a Master degree in de-sign of telecommunication systems from ParisTech Tlcom, France. Since 1981 he has been with the Department of Electrical Engineering (ESAT) of the KU Leuven, where he has been involved in research on microwave antennas, passive components, interconnects, microwave integrated circuits and MMICs, linear and non-linear device modelling, MEMS and wireless communications. He is former chair of IEEE AP/MTT-Benelux and past chair of URSI-Benelux. Bart Nauwelaers teaches courses on microwave engineering, on analogue and digital communications, on wireless communications and on design in electronics and telecommunications. For the last twenty years he has served education in several functions, the last one being program director for the bachelor and master programs in electrical engineering.

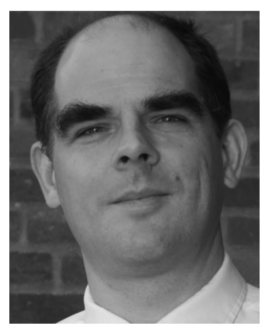

Renaud Gillon (S'89-M'92) was born in Brus-sels, Belgium, in 1968. He received the M.Sc. and Ph.D. degrees in electrical engineering from the Uni-versite catholique de Louvain, Louvain-la-Neuve, Belgium, in 1991 and 1998, respectively. In 1998, he joined ON Semiconductor Belgium (ONSEMI), Oudenaarde, Belgium (then Alcatel Microelectron-ics) as a Modelling Engineer and managed the team from 2000 to early 2006, taking an active role in the development of design kits for the company's RF and smart-power processes. He is currently respon- sible for several research and innovation programs at ONSEMI, in the field of ESD, EMC and power. His current research interests include modelling of HV and RF components, RF measurement techniques, electro-thermal simulation and predictive simulation of EMC tests at the IC level. He is the author or co-author of more than 60 publications.

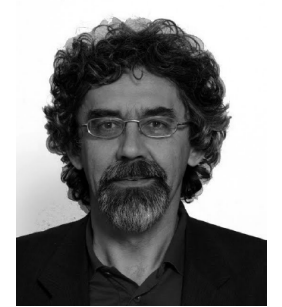

Adrijan Baric (M'98-SM'16) received the Dipl.-Ing. and M.Sc. degrees in electrical engineering from the University of Zagreb, Zagreb, Croatia, in 1982 and 1985, respectively, and the Ph.D. degree in electronics from the Dublin City University, Dublin, Ireland, in 1995. Since 1984, he has been with the University of Zagreb, where he is currently a Pro-fessor. His research interests include semiconductor device modeling, integrated circuit design, intercon-nect modeling and electromagnetic compatibility. 